\title{
OFDM Signaling for SWIPT Systems under High Power Amplifier Nonlinearities and Memory Effects
}

\author{
Souhir Lajnef, Konstantinos Ntougias, and Ioannis Krikidis \\ Department of Electrical and Computer Engineering, University of Cyprus \\ Email: \{lajnef.souhir, ntougias.konstantinos, krikidis.ioannis\}@ucy.ac.cy
}

\begin{abstract}
In this paper, we study the employment of conventional orthogonal frequency division multiplexing (OFDM) signals in simultaneous wireless information and power transfer (SWIPT) systems. Specifically, we investigate the impact of high peak-to-average power ratio (PAPR) on OFDM-based information/energy transfer under the nonlinearities and memory effects introduced by high power amplifier (HPA) at the transmitter. A closed-form expression of the symbol error rate (SER) as a function of the PAPR is derived for a Gaussian channel in this context. We notice that even under these realistic non-idealities, PAPR deteriorates the SER performance while facilitating energy transfer efficiency, as expected. To further enlarge the rate-energy (R-E) region, a predistortion scheme that allows the transmission of unclipped high PAPR OFDM signals is also proposed. Numerical simulations based on MATLAB and ADS software validate the impact of PAPR in SWIPT systems, regarding both the information detection and energy harvesting processes.

Index Terms-Orthogonal frequency division multiplexing (OFDM), high power amplifier (HPA), peak-to-average power ratio (PAPR), simultaneous wireless information and power transfer (SWIPT), predistortion.
\end{abstract}

\section{INTRODUCTION}

The number of energy-constrained wireless communication devices, such as wireless sensors and smartphones, is constantly increasing over the last decade [1]. This trend is expected to continue with an even more abrupt pace in the foreseeable future, thanks to the emergence of the massive Internet-of-Things (IoT) paradigm [2], as well as due to the continuous drop of the power consumption required for performing specific tasks according to Koomey's law [3]. The high volume of devices with limited energy storage capacity, along with the fact that these nodes are often installed at hard-to-reach areas (e.g., industrial plants), render the wired charging or replacement of their batteries a challenging and costly endeavor. Introducing energy harvesting $(\mathrm{EH})$ capabilities at these low-power devices has been recently proposed as a means to address this issue. This concept refers to the capturing of energy from natural or man-made sources in the surrounding environment; its conversion into electrical energy; and its storage for future use, in order to prolong the lifetime of these devices. Wireless power transfer (WPT) refers to the use of dedicated radio frequency (RF) energy sources, in order to achieve stable and controllable supply of RF power for EH purposes. This disruptive technology presents long range, inherently supports user mobility and quality-ofservice (QoS) provisioning, and allows for energy multicasting (i.e., one-to-many wireless charging). The integration of WPT with wireless information transfer (WIT), which is referred to as wireless powered communication (WPC), makes possible the realization of the envisioned next-generation energysustainable wireless communication systems. However, the transition of this vision from its conceptual state into practical implementation requires a re-design of the involved waveforms, communication protocols, receiver architectures, etc., as well as the definition of novel performance metrics, the study of new performance trade-offs, and the derivation of relevant performance bounds [4].

Simultaneous wireless information and power transfer (SWIPT) corresponds to a WPC variant, where information and power are concurrently transferred in the downlink (DL) from one or more access points (AP) to one or multiple user equipment (UE) devices [5]. This approach is characterized by a fundamental information rate-energy trade-off or rateenergy (R-E) region, which turns the design, optimization, and performance evaluation of SWIPT techniques into a highly non-trivial task. Typically, a UE integrates an information decoding (ID) circuit and an EH circuit into a single receiver. The main receiver architectures that are commonly adopted in practice are time-switching (TS), where the UE switches in time between ID and EH operation, and power splitting (PS), where the received RF signal is split in the power domain into two streams, one that is sent to the ID circuit for data detection and another that is directed to the $\mathrm{EH}$ circuit for conversion into a direct-current (DC) voltage and energy storage. Another strategy is the use of separate EH and ID receivers.

Numerous research efforts have been devoted to waveform design for SWIPT in the literature. The authors in [6] assume the use of PS at the UEs and propose a waveform that is comprised by the superposition of a deterministic multi-sine power waveform and an orthogonal frequency division multiplexing (OFDM) information waveform. However, this superposition method causes undue power consumption. The utilization of multi-sine waveforms for energy transfer and the use of their distinct high peak-to-average power ratio (PAPR) for information transmission is considered in [7]. This technique achieves higher power conversion efficiency at the receiver while requiring less energy and low complexity for ID, but it provides lower data rate. Other research works focus on the use of multi-carrier modulated waveforms (e.g., OFDM) for both information and power transfer purposes. In [8], an OFDMbased multi-user broadcast SWIPT system is considered. The goal of OFDM-based SWIPT in this paper is to enhance 
the R-E region. In this work, two transmission schemes are investigated, namely, time-division multiple access (TDMA) based information transmission with TS UEs and OFDMAbased information transmission with PS UEs. In order to minimize the complexity related with the implementation of TS or PS, the authors in [9] exploit the cyclic prefix (CP) portion of the OFDM symbols for $\mathrm{EH}$.

Another line of research relies on the observation, derived both analytically and experimentally, that signals featuring a high PAPR, such as OFDM, white noise, and chaotic signals, can provide high DC output voltage and RF-DC conversion efficiency improvement when compared to continuous waveform signals [10], [11]. On the other hand, high PAPR signals are typically avoided in communication systems, since they result in nonlinear distortions and clipping caused by the nonlinearity and the "memory effect" of the high power amplifier (HPA) at the transmitter [12]. Therefore, we should strike a desirable balance between the linearity of the HPA, to suppress the transmit distortion that deteriorates the performance of information transfer, and its efficiency, to boost the performance of WPT.

In our previous work [13], we investigated the effect of practical HPA impairments in SWIPT performance. More specifically, we characterized the achievable R-E region of the considered MIMO SWIPT system and we demonstrated that the HPA significantly degrades SWIPT performance. In this work, we extend our design framework by studying the impact of nonlinear distortions and memory effects attributed to the HPA on OFDM-based SWIPT. Furthermore, we optimize the OFDM waveform in the presence of HPA distortions by utilizing a predistortion scheme at the transmitter side with low input back-off (IBO) to enhance the efficiency of the HPA and enable the transmission of unclipped high PAPR signals [14]. This method allows us to control the PAPR level. In addition, we derive the expression of the symbol error rate (SER) as a function of the PAPR. This enables us to achieve the desired level of PAPR via predistortion, based on the information transfer and energy transfer requirements. Numerical simulations highlight the effect of PAPR and predistortion on the information (SER) and power (harvested power) transfer.

\section{SySTEM MODEL}

We consider a SWIPT system, where power and information are transmitted simultaneously by using an OFDM waveform, as shown in Fig. 1. Specifically, the transmitter sends an OFDM signal to an ID receiver and an $\mathrm{EH}$ receiver. The HPA at the transmitter is characterized by non-linearities. Also, we apply predistortion at the transmitter to improve the performance.

\section{A. Transmitted Signal}

The general architecture of an OFDM transmitter is shown in Fig. 1. The input bits are mapped onto appropriate symbols, based on the modulation used on the corresponding subcarrier. Then, the modulation symbols are grouped and processed by the inverse discrete Fourier transform (IDFT) module. The parallel output of the IDFT is converted into a serial stream of OFDM symbols and then converted into an analog baseband signal by using a digital-to-analog converter (ADC). Finally, the signal is linearized by using a predistortion (PD) unit, which is installed right before the HPA. The complex baseband representation of the OFDM signal with $N$ subcarriers is given by

$$
x(n)=\frac{1}{N} \sum_{k=0}^{N-1} s_{k} e^{j 2 k n \pi / N}, \quad n=0,1, \ldots, N-1,
$$

where $s_{k}$ are the complex data symbols, $k$ denotes the subcarrier index, and $n$ represents the discrete time domain index. We assume that the data symbols $s_{k}$ are independent and identically distributed (i.i.d.) with zero mean and variance $V$. By the central limit theorem (CLT), $x(n)$ can be assumed to be a complex Gaussian process with zero mean and variance $P_{\text {avg }}=\frac{V}{N}$, when $N$ is large [15].

The HPA output differs from the ideal HPA response due to the presence of nonlinearities and memory effects that severely degrade the system performance [16]. In order to characterize the memory effects of the nonlinear HPA, we use the memory polynomial model (MPM) [14],

$$
x_{\text {out }}(n)=\sum_{p=1}^{P} \sum_{m=0}^{M} c_{p, m} x_{\text {in }}(n-m)\left|x_{\text {in }}(n-m)\right|^{p-1},
$$

where $P$ refers to the non-linearity order, $M$ corresponds to the memory depth, and $c_{p, m}$ with $p=1, \cdots, P, m=0, \cdots, M$, denotes the complex MPM coefficients. The memory depth $(M)$ implies that $x_{\text {out }}(n)$ depends on the past $M$ input samples, i.e, $x_{\text {out }}(n)=f\left(x_{\text {in }}(n), \cdots, x_{\text {in }}(n-M)\right)$. Accordingly, (2) can be written in a generic form as $x_{\text {out }}(n)=\Phi \mathrm{C}_{\mathrm{HPA}}$, where

$$
\boldsymbol{\Phi}=\left[\begin{array}{c}
x_{\text {in }}(n) \\
\vdots \\
x_{\text {in }}(n-M) \\
x_{\text {in }}(n)\left|x_{\text {in }}(n)\right| \\
\vdots \\
x_{\text {in }}(n-M)\left|x_{\text {in }}(n-M)\right| \\
\vdots \\
x_{\text {in }}(n-M)\left|x_{\text {in }}(n-M)\right|^{P-1}
\end{array}\right]^{T},
$$

and $\mathrm{C}_{\mathrm{HPA}}=\left[c_{1,0}, \cdots, c_{1, M}, c_{2,0}, \cdots, c_{2, M}, \cdots, c_{P, M}\right]^{T}$. For a given set of input-output sample pairs, we recursively obtain $\mathrm{C}_{\mathrm{HPA}}$ by solving an over-determined system of equations using the least squares (LS) method [17]. The predistortion function is equivalent to the behavioral modeling of the HPA (MPM) reverse function obtained by swapping the HPA input and output signals with appropriate small signal gain normalization [14]. So in order to identify the predistortion, we are restricted by an intermediate step, in which the HPA is first identified, and its model is then used for the extraction of predistortion coefficients. 


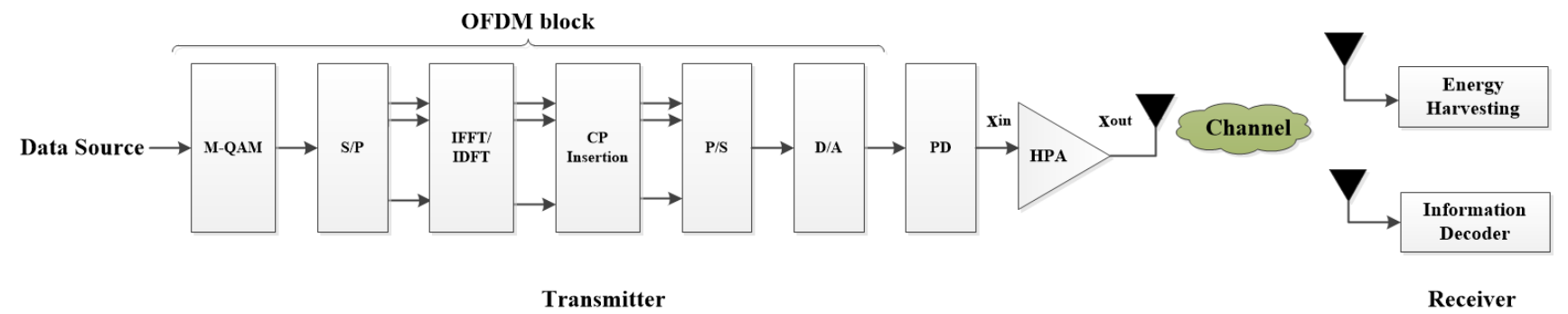

Fig. 1: A SWIPT system with a separate EH receiver and an ID receiver.

\section{B. Received Signal}

The received complex data symbol at the $k$-th subcarrier obtained after discrete Fourier transform (DFT), assuming transmission over an additive white Gaussian noise (AWGN) channel, is given by [18]

$$
\hat{s}_{k}=\sum_{n=0}^{N-1} x_{\text {out }}(n) e^{-j 2 k n \pi / N}+n_{0}(k),
$$

where $n_{0}(k)$ is the corresponding AWGN noise in the $k$-th subcarrier with zero mean and variance $N_{0}$. By substituting (2) into (3) we obtain

$$
\begin{gathered}
\hat{s}_{k}=\sum_{n=0}^{N-1} \sum_{p=1}^{P} \sum_{m=0}^{M} c_{p, m} x_{\mathrm{in}}(n-m)\left|x_{\mathrm{in}}(n-m)\right|^{p-1} \\
e^{-j 2 k n \pi / N}+n_{0}(k)=\sum_{m=0}^{M} \hat{y}_{m}(k)+n_{0}(k) .
\end{gathered}
$$

For convenience and without loss of generality, we assume that $P=3$ and consider only the odd order nonlinearities, since the even terms generate intermodulation products far away from the input channels which can be filtered. Under this context, we obtain

$$
\begin{aligned}
& \hat{y}_{m}(k)=\sum_{n=0}^{N-1} c_{1, m} x_{\mathrm{in}}(n-m) e^{-j 2 k n \pi / N} \\
& +\sum_{n=0}^{N-1} c_{3, m} x_{\mathrm{in}}(n-m)\left|x_{\mathrm{in}}(n-m)\right|^{2} e^{-j 2 k n \pi / N} .
\end{aligned}
$$

After some mathematical manipulations and by using the circular time shift property of the DFT [19], the expression (5) can be written as

$$
\begin{aligned}
\hat{y}_{m}(k)= & \sum_{n=0}^{N-1} c_{1, m} x(n) e^{-j 2 k n m \pi / N} e^{-j 2 k n \pi / N} \\
& +\sum_{n=0}^{N-1} c_{3, m} x(n)|x(n)|^{2} e^{-j 2 k m \pi / N} e^{-j 2 k n \pi / N} \\
& =s_{k} \beta_{m}(k)+\gamma_{m}(k),
\end{aligned}
$$

where

$$
\beta_{m}(k)=c_{1, m} e^{-j 2 k m \pi / N}+\frac{c_{3, m} e^{-j 2 k m \pi / N}}{N} \sum_{n=0}^{N-1}|x(n)|^{2},
$$

$\gamma_{m}(k)=\frac{c_{3, m} e^{-j 2 k m \pi / N}}{N} \sum_{l=0}^{N-1} s_{l} \sum_{n=0}^{N-1}|x(n)|^{2} e^{j 2 k n \pi(-k+l) / N}$.

Thus, the received symbol obtained after DFT processing can be written as

$$
\hat{s}_{k}=s_{k} \beta(k)+\gamma(k)+n_{0}(k),
$$

where

$$
\beta(k)=\sum_{m=0}^{M} \beta_{m}(k) \quad \text { and } \quad \gamma(k)=\sum_{m=0}^{M} \gamma_{m}(k) .
$$

$\beta(k)$ represents the complex attenuation component, which is attributed mainly to the distortions of the HPA. The amount of distortion depends upon the coefficients of the MPM and the power of the OFDM symbol as well as on the subcarriers' position. The term $\gamma(k)$ represents the nonlinear noise component.

\section{Symbol ERror Rate (SER) AND}

Peak-To-Average-Power-Ratio (PAPR) Analysis

In this section, the effect of the nonlinearity distortion is analyzed based on the expression of the SER for each subcarrier, considering transmission over an AWGN channel of M-QAM (quadrature amplitude modulation) signals [19]. Under this context, the SER of subcarrier $k$ is given by

$$
\operatorname{SER}(k)=2\left(1-\frac{1}{\sqrt{M}}\right) \mathrm{Q}\left(\sqrt{\frac{3 \operatorname{SNR}(k)}{M-1}}\right),
$$

where $Q(\cdot)$ denotes the Gaussian tail function. The received signal-to-noise-ratio (SNR) for each subcarrier at the output of the DFT unit depends on the working point of the HPA and it can be expressed as

$$
\operatorname{SNR}(k)=\frac{V E\left[|\beta(k)|^{2}\right]}{E\left[|\gamma(k)|^{2}\right]+N_{0}},
$$

where $E[\cdot]$ denotes the expectation operator.

We define the IBO, which is used to quantify the nonlinear distortion incuded by the HPA, as the ratio between input saturation power threshold and average power, i.e., $\mathrm{IBO}=P_{\text {sat }} / P_{\text {avg }}$. Hence, (11) and (12) show us the impact of both $P_{\mathrm{avg}}=V / N$ and IBO on the received SER and SNR, 
respectively. Since the IBO is inversely related to $P_{\text {avg }}$, as we increase the IBO we decrease the HPA efficiency.

The PAPR of an OFDM signal can be defined as

$$
\text { PAPR }=\frac{\max |x(n)|^{2}}{P_{\text {avg }}}=\frac{P_{\max }}{P_{\text {avg }}} .
$$

Ideally, the transmitter should be able of sending an unclipped signal, in order to maintain its high PAPR which enhances the RF-DC conversion efficiency in the rectifier circuit [16]. Thus, controlling the PAPR by setting the average power to a level that is just low enough to minimize the problem of clipping is of high interest. In order to maintain an efficient trade-off between linearity and power efficiency, it is best to drive to HPA to a sufficiently power efficient regime with an appropriate value of IBO and retain a tolerable level of nonlinear distortion. Predistortion can be used to this end, in order to improve the efficiency of the HPA and reduce the nonlinear distortions.

The relation between PAPR and the error vector magnitude (EVM), which is a performance metric for assessing the quality of communication, for a known HPA operating point back-off can be expressed as follows:

$$
\text { PAPR }=10 \log \left(10^{\frac{\mathrm{IBO}}{10}}+10^{\frac{\mathrm{EVM}}{10}}\right) .
$$

Larger back-off values increase the corresponding tolerable PAPR for the same EVM value.

The distortion metric due to the PAPR issue is usually expressed in terms of the EVM. The relationship between EVM and SER based on the EVM estimation from BER [20] is given as

$$
\operatorname{EVM}(\mathrm{SER})=\sqrt{\frac{3}{M-1}} \frac{1}{\left[\mathrm{Q}^{-1}\left(\frac{\mathrm{SER}}{4}\right)\right]} .
$$

Finally, the relation between PAPR (in $\mathrm{dB}$ ) and SER can be obtained by substituting (15) into (14). Thus, we have

$$
\operatorname{SER}(\mathrm{PAPR})=4 \mathrm{Q}\left[\frac{\sqrt{\frac{3}{M-1}}}{10 \log \left(10^{\mathrm{PAPR} / 10}-10^{\mathrm{IBO} / 10}\right)}\right] \text {. }
$$

By setting the PAPR level at an appropriate value based on (16) via the PD unit, we can ensure that a sufficiently high amount of power is harvested with minimal information transfer performance degradation.

\section{Simulations Results}

\section{A. Matlab Simulations}

This section aims at assessing the impact of the HPA nonlinearity with memory on the SER performance and the PAPR estimation without/with the use of a predistortion scheme via computer simulations. We investigate the impact of the varying system parameters such as the PAPR and the IBO on the performance of SWIPT systems.

First, we focus on the distribution of symbol errors as a function of PAPR for various IBOs, as depicted in Fig. 2. It can be noticed that the errors are densely accumulated in the

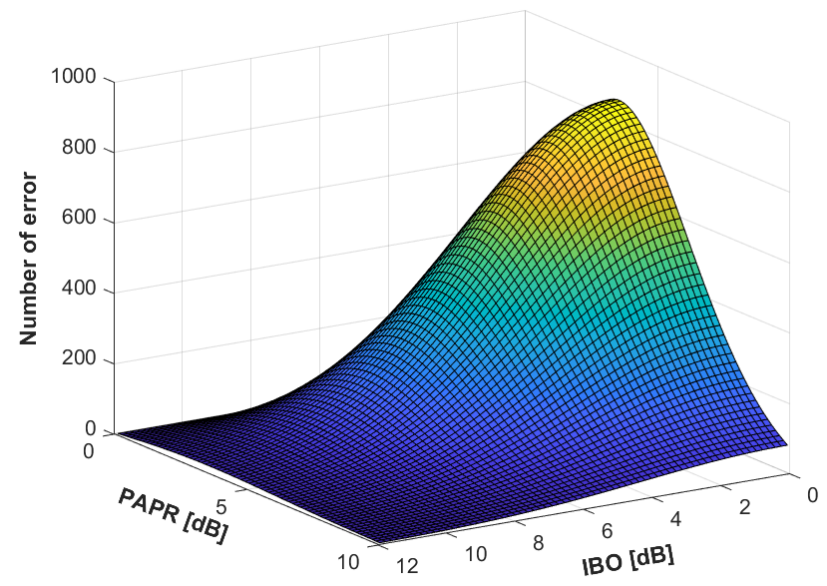

Fig. 2: Error distribution versus PAPR for various IBO values.

PAPR range between $3 \mathrm{~dB}$ and $9 \mathrm{~dB}$ for low IBO values. Hence, the high PAPR of the OFDM signal requires large power back-off for the linear regime operation of the HPA, incurring high loss in power efficiency.

Fig. 9 presents the average SER performance versus the SNR for various values of IBO. Nonlinear HPA with memory affects each subcarrier differently. As it can be seen from the plot, the SER performance increases with the increase of the IBO. Nevertheless, by operating the HPA at a higher back-off leads to a power efficiency loss in the HPA. The application of a predistortion scheme improves the power efficiency of the OFDM transmitter significantly by alleviating the requirement for high power back-off.

SER performance versus varying levels of PAPR is illustrated in Fig. 9. It is observed that the SER increases with the PAPR until the saturation region. Hence, a trade-off between high PAPR for energy transfer efficiency improvement and low PAPR for information transfer with minimal distortion is required. Fig. 5 focuses on the employment of a predistortion scheme at the transmitter side. First, we evaluate the accuracy in the frequency domain; the power spectrum confirms the spectral regrowth caused by the HPA nonlinearity and the memory effects. The figure also demonstrates that the outof-band distortion has been reduced by more than $15 \mathrm{~dB}$ due to the predistortion. Even though the predistortion is used to compensate for the HPA nonlinearities, some amount of IBO is required to remove nonlinear distortion completely, since the PAPR is high.

Another metric that is considered to evaluate the system performance is the total degradation (TD). The TD is a performance measure that describes the difference between the maximum power of the HPA and the output power of a linear amplifier required to assure a predefined SER. The TD as a function of IBO is given by

$$
\mathrm{TD}(\mathrm{dB})=(\mathrm{SNR})_{\mathrm{NL}}+(\mathrm{SNR})_{\mathrm{Lin}}+\mathrm{IBO},
$$

where $\mathrm{SNR}_{\mathrm{NL}}$ and $\mathrm{SNR}_{\mathrm{Lin}}$ are the required $\mathrm{SNR}$ values for 

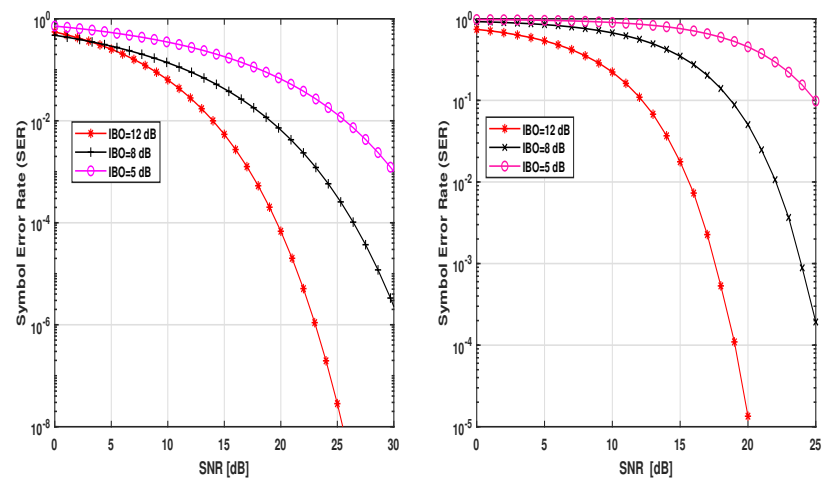

Fig. 3: SER performance with 16-QAM and $N=512$ for different values of IBO without (left)/with (right) predistortion.

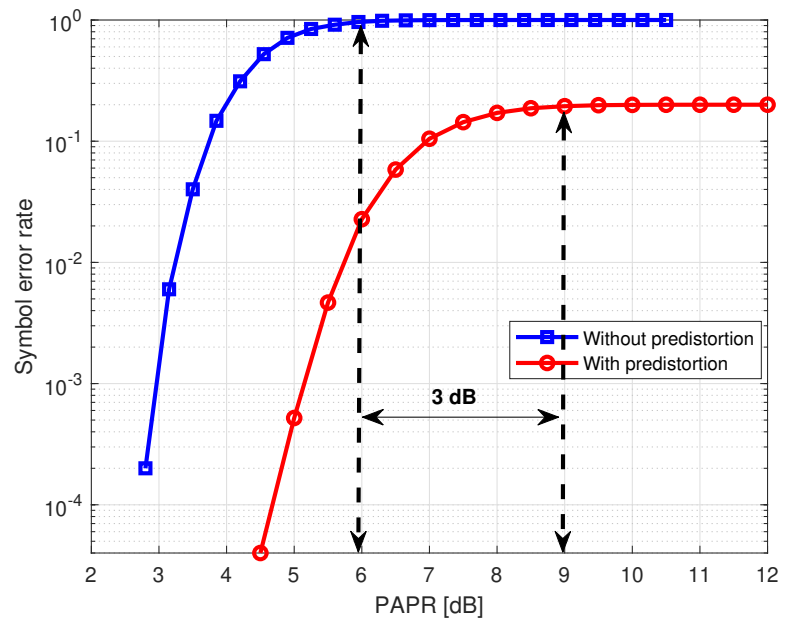

Fig. 4: Symbol error rate versus PAPR.

the nonlinear and the linear amplifier, respectively, which reflect the loss in SNR of a practical HPA compared to an ideal HPA that achieves the same SER. The TD performance versus IBO with a predefined SER of $10^{-4}$ is presented in Fig. 6. As shown, the optimal IBO value corresponds to the minimum of each curve. Predistortion is effective in providing a substantial TD reduction of about $3 \mathrm{~dB}$ and improving the power efficiency (optimum IBO) by about $5.4 \mathrm{~dB}$.

\section{B. ADS Simulations}

WPT is an important building block of SWIPT, and the extension of the R-E region of SWIPT systems relies heavily on the efficiency of WPT-and, therefore, on waveform design. The objective of this section is to explore the effects of the main transmitted OFDM waveform's attributes, specifically the PAPR level of the signal, on the received DC power level. To this end, we used Advanced Design System (ADS) software to study the impact of high-PAPR OFDM waveforms on RF-DC conversion efficiency. As shown in Fig. 7, the ADS schematic is composed of an input OFDM source playing the role of the receive antenna, a matching network, a rectifier circuit, a
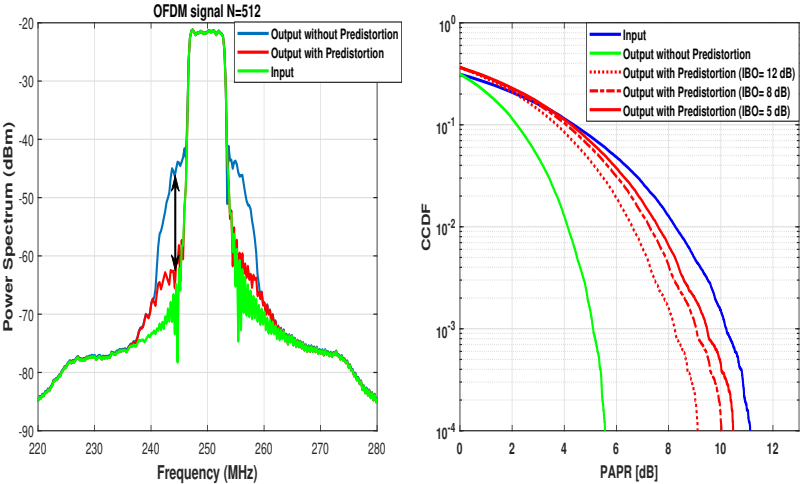

Fig. 5: Effect of predistortion on the HPA output signal

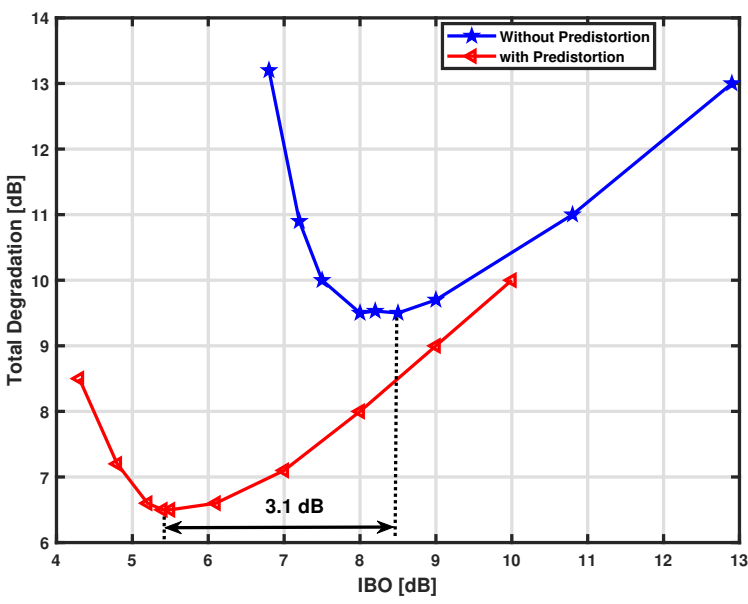

Fig. 6: Total Degradation.

low-pass filter and a load. The rectifier is comprised by one or more diodes in some arrangement.

The diodes are a key source of power losses. Furthermore, their use results in a non-linear input-output rectifier characteristic. The simplest rectifier topology is the single serial connection, which is characterized by high efficiency in the low input power regime. The role of the matching circuit is to match the antenna impedance with the input impedance of the rectifier, in order to reduce the reflection loss and ensure maximum power transfer. The input impedance is dependent on the operating frequency, the RF input power and the load resistance. The matching network performance is evaluated through the simulation of the input reflection coefficient $S_{11}$. In Fig. 8, the Smith Chart shows the input impedance $Z_{i n}$ as a function of the operating frequency, which in this example is set at $2.45 \mathrm{GHz}$.

The power conversion efficiency is not only a function of the rectifier design but also depends on the input waveform. Thereby, the exploitation of OFDM signals with a varying envelope leads to a higher WPT performance. On the one hand, signals with a high PAPR level could play an important role in WPT allowing to overcome the nonlinearity of the 


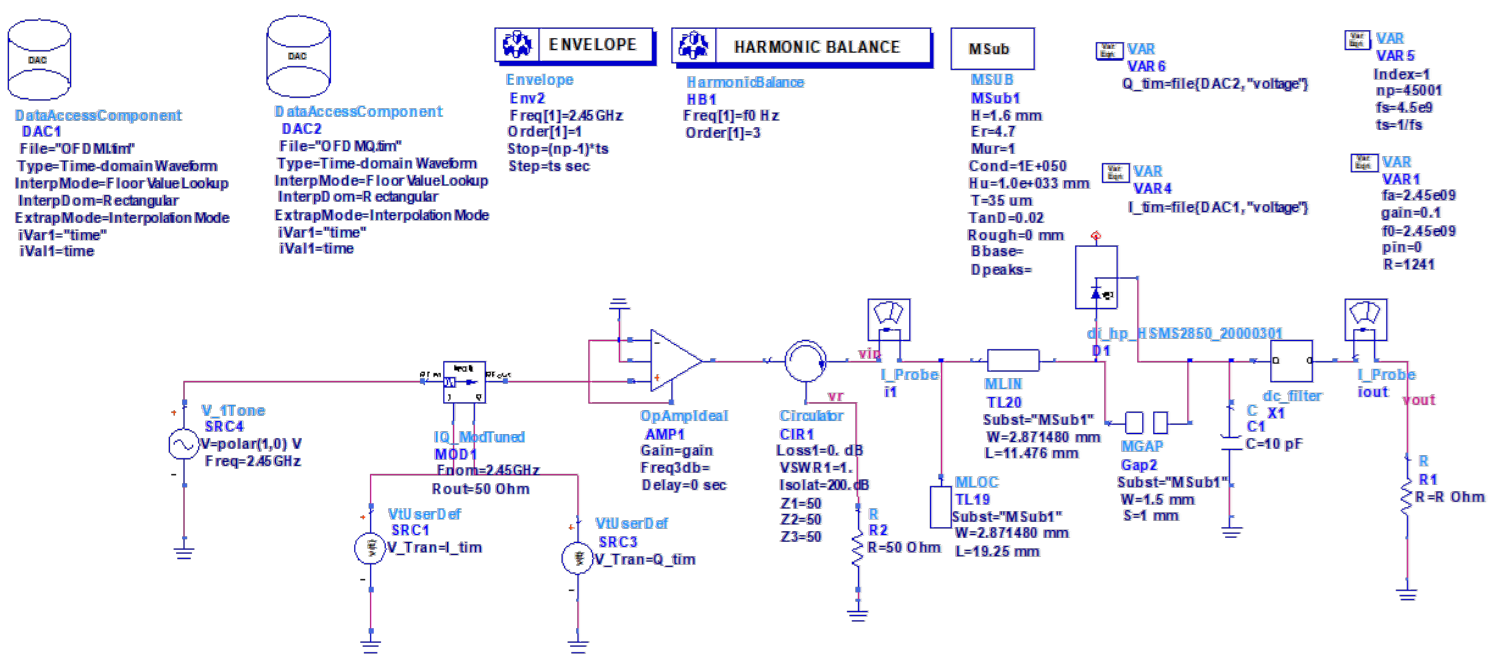

Fig. 7: ADS schematic of single serial diode topology excited by OFDM signal
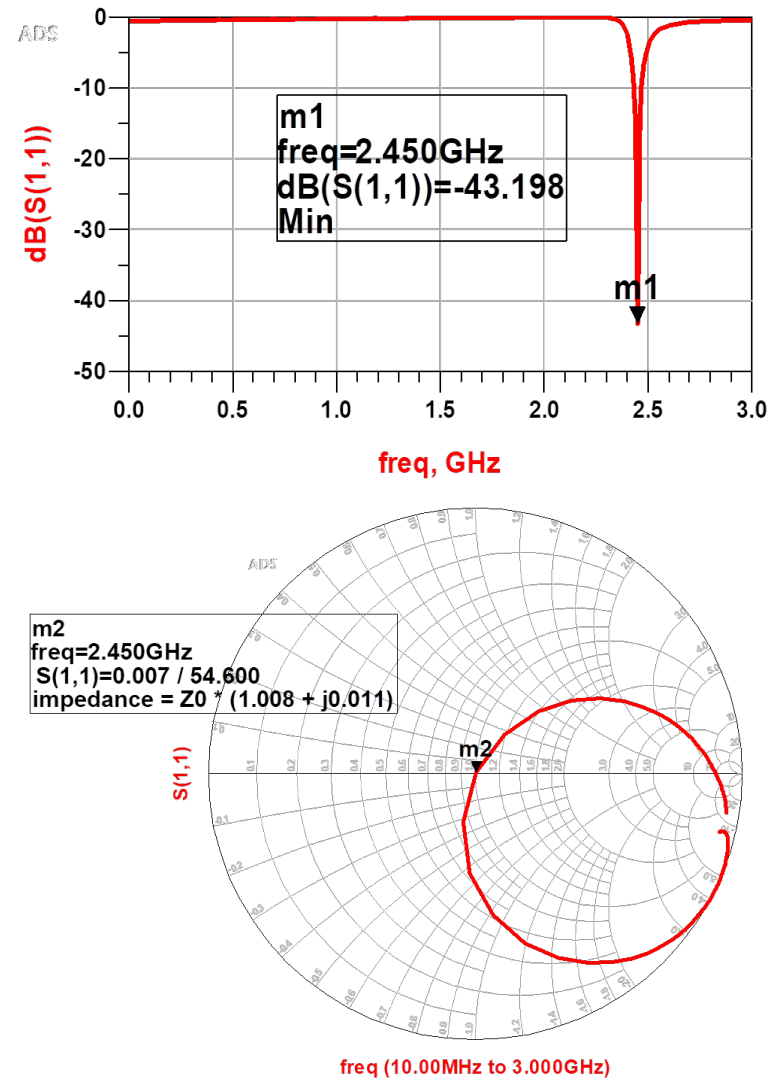

Fig. 8: Design of $2.45 \mathrm{GHz}$ matching network.

rectifier and the problems with rectification and conversion of weak signals. On the other hand, the usage of high PAPR signals requires an HPA with a large back-off, thus decreasing the power efficiency. The RF-DC conversion efficiency drops when the PAPR level is above $9 \mathrm{~dB}$ as shown in Fig. 10. It is worth noting that the efficiency is not a monotonically

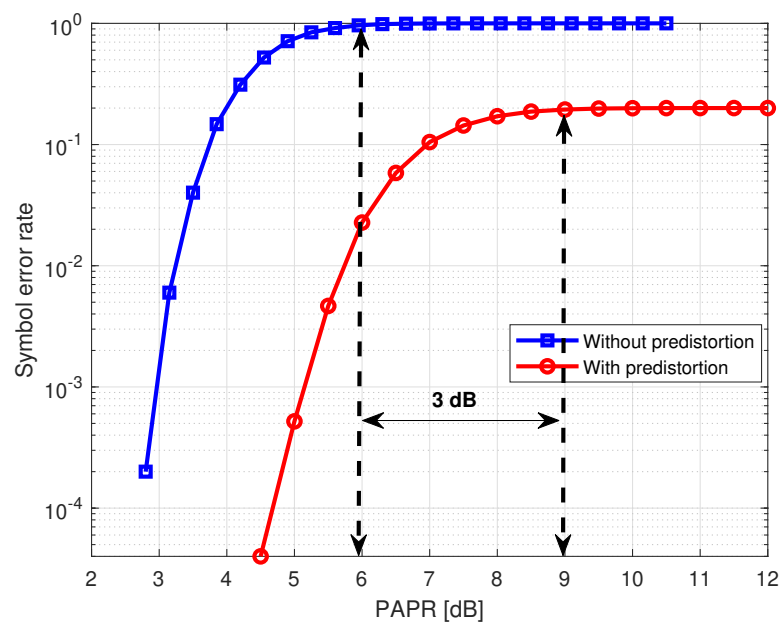

Fig. 9: Symbol error rate versus PAPR.

increasing function and the increase of the PAPR cause a sharp decrease in efficiency. However, exciting the circuit by the predistorted OFDM signal, shows that the employment of the predistortion scheme is efficient to keep high PAPR level till some threshold higher than $9 \mathrm{~dB}$ and can still provide a converted voltage with efficiency up to $50 \%$.

Thus, if we aim at high EH efficiency, a waveform with higher PAPR performs better than the one with lower PAPR, while if we focus on information decoding, the waveform with the highest PAPR performs the worst. With this regard, controlling the PAPR is of interest as an important step in improving the rate-energy trade-off in SWIPT systems.

\section{CONClusion}

In this work, we have studied the impact of high-PAPR OFDM signals on SWIPT performance in the presence of nonlinear distortion and memory effects attributed to the HPA. 

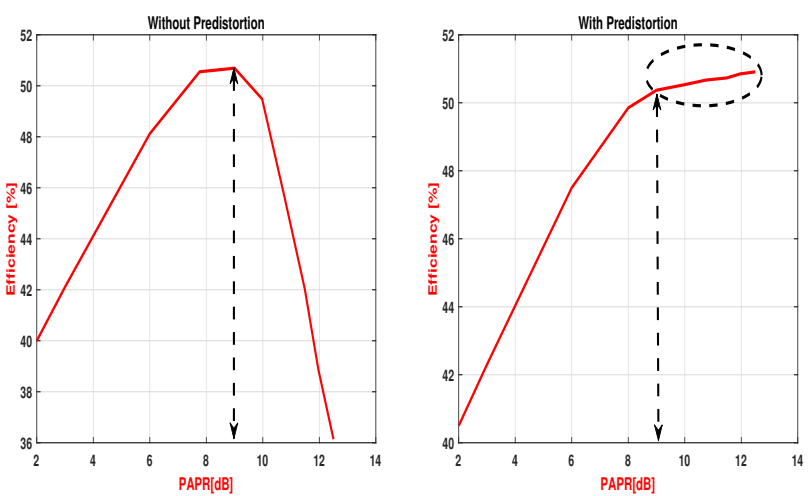

Fig. 10: Effect of predistortion on the HPA output signal

First, we derived a closed-form expression for the SER as a function of the PAPR. This formula can be used as a design guideline for the waveform, according to the required SER performance. On the other hand, we demonstrated via computer simulations that the transmit HPA efficiency decreases as the PAPR of the transmit waveform increases and we highlighted the fact that the use of predistortion improves the performance of energy transfer while balancing the degradation in information transfer performance.

\section{ACKNOWLEDGMENT}

This work has received funding from the European Research Council (ERC) under the European Union's Horizon 2020 research and innovation programme(Grant agreement No. 819819). It is also co-funded by the European Regional Development Fund and the Republic of Cyprus through the Research and Innovation Foundation, under the projects INFRASTRUCTURES/1216/0017 (IRIDA) and EXCELLENCE/0918/0377 (PRIME).

\section{REFERENCES}

[1] Ericsson, "Mobility Report," Nov. 2019.

[2] J. Navvaro-Ortiz et al., "A Survey on 5G Usage Scenarios and Traffic Models," IEEE Commun. Surv. and Tut., vol. 22, no. 2, pp. 905-929, 2020, secondquarter.

[3] J. G. Koomey, S. Berard, M. Sanchez, and H. Wong, "Implications of Historical Trends in the Electrical Efficiency of Computing," IEEE Ann. History Comput., vol. 33, no. 3, pp. 46-54, Mar. 2011.

[4] B. Clerckx, R. Zhang, R. Schober, D. W. K. Ng, D. I. Kim, and H. V. Poor, "Fundamentals of Wireless Information and Power Transfer: From RF Energy Harvester Models to Signal and System Designs," IEEE Journal on Selected Areas in Communications, vol. 37, no. 1, pp. 433, 2019.

[5] I. Krikidis, S. Timotheou, S. Nikolaou, G. Zheng, D. W. K. Ng, and R. Schober, "Simultaneous wireless information and power transfer in modern communication systems," IEEE Commun. Mag., vol. 52, no. 11, pp. 104-110, Nov. 2014.

[6] B. Clerckx, R. Zhang, R. Schober, D. W. K. Ng, D. I. Kim, and H. V. Poor, "Wireless information and power transfer: Nonlinearity, waveform design, and rate-energy tradeoff," IEEE Trans. on Signal Processing, vol. 66, no. 4, pp. 847-862, Nov. 2017.

[7] D. I. Kim, J. H. Moon, and J. J. Park, "New SWIPT using PAPR: How it works," IEEE Wireless Commun. Lett., vol. 5, no. 6, pp. 672-675, Dec. 2016.

[8] X. Zhou, R. Zhang, and C. K. Ho, "Wireless information and power transfer in multiuser ofdm systems," IEEE Trans. Wireless Commun., vol. 13, no. 4, pp. 2282-2294, Apr. 2014.
[9] M. Maso, S. Lakshminarayana, and T. Q. S. Quek, "Adaptive selfsustainable ofdm communications," Proc. IEEE Intl. Conf. on Commu., vol. 15, pp. 4662-4668, Jun. 2014.

[10] R. Scheeler, S. Korhummel, and G. D. Popovic, "Daul-frequency ultra low-power efficient $0.5 \mathrm{~g}$ rectenna,", IEEE Microwave Mag, vol. 63, no. 5 , pp. $1758-1767$, May. 2015.

[11] B. Clerckx, R. Zhang, R. Schober, D. W. K. Ng, D. I. Kim, and H. V. Poor, "Fundamentals of wireless information and power transfer: From $\mathrm{RF}$ energy harvester models to signal and system designs," IEEE $J$. Selec. Areas Commun., vol. 37, no. 1, pp. 4-33, Jan. 2019.

[12] S. H. Han and J. H. Lee, "An overview of peak-to-average power ratio reduction techniques for multicarrier transmission," IEEE Trans. Wireless Commun., vol. 12, no. 2, pp. 56-65, Apr. 2005.

[13] P. mukherjee, S. Lajnef, and I. Krikidis, "Mimo swipt systems with power amplifier nonlinearities and memory effects," IEEE Wireless Commun. Lett., pp. 1-1, Aug. 2020.

[14] J. Kim and K. Konstantinou, "Digital predistortion of wideband signals based on power amplifier model with memory," Electron. Letter, vol. 37, no. 23, pp. 1417-1418, Nov. 2001.

[15] H. Ochiai and H. Imai, "On the distribution of the peak-to-average power ratio in ofdm signals," IEEE Trans. on Commun., vol. 49, no. 2, pp. 282-289, Feb. 2001.

[16] C. R. Valenta, M. M. Morys, and Z. Durgin, "Theoretical energyconversion efficiency for energy-harvesting circuits under power optimized waveform excitation," IEEE Trans. Microw. Theory Techn., vol. 15, no. 1, pp. 109-114, 2014.

[17] F. M. Ghannouchi, O. Hammi, and M. Helaoui, Behavioral modeling and predistortion of wideband wireless transmitters. John Wiley \& Sons Ltd, 2015.

[18] A. Ruiz, J. M. Cioffi, and S. Kasturia, "Discrete multiple tone modulation with coset coding for the spectrally shaped channel," IEEE Trans. Commun., vol. 33, pp. 665-675, June 1992.

[19] J. G. Proakis, Digital Communications. New York : McGraw-Hill, 1995.

[20] A. Lipovac, B. Modlic, and M. Grgić, "Ofdm error floor based evm estimation," 2016, p. 1. 